\title{
Flu vaccine safe for egg allergic children
}

Published online: 26 October 2012

(C) Springer Healthcare 2012

medwireNews: Trivalent seasonal influenza vaccine can be given safely to children with a history of severe egg allergy, say researchers.

The findings may help ease fears about giving the vaccination, which is grown in embryonated chicken eggs and contains residual amounts of the major egg allergen ovalbumin, to children with such allergies for fear it might trigger a reaction.

The team, led by Matthew Greenhawt (University of Michigan, Ann Arbor, USA), carried out a two-phase, multicenter study between October 2010 and March 2012. The study included 31 children with severe egg allergy, 14 of whom were assigned to receive influenza vaccine (group A) and 17 placebo (group B).

In the first stage of the study, the children in group A received o.1 mL influenza vaccine followed by a 30 minute break. If no adverse reaction occurred then the rest of an age appropriate dose was given to the children. The children in group B were given a small dose of placebo, then the full age appropriate vaccine dose 30 minutes later.

The second stage of the study compared patients given a split dose of vaccine $(n=25)$ to those with allergy who elected to have a single dose $(n=87)$.

Despite the fact that $45.1 \%$ of participants had a history of anaphylaxis after egg ingestion in phase one and $77.6 \%$ in phase two, none of the children receiving the vaccine had an allergic reaction in either phase of the study.

"Current Advisory Committee on Immunization Practices and American Academy of Pediatrics recommendations state that egg allergic children [EAC] with severe allergy (ie, those having more than hives only after egg ingestion) should be referred to an allergy specialist for further evaluation before influenza vaccination," explain Greenhawt and co-authors.

"However, these children tolerate the influenza vaccine, and allergy referral poses a barrier to their being vaccinated," they add.

"Thus, it would seem that EAC with allergy of any severity can be safely vaccinated in a primary care setting, with the only precaution being they be observed for 30 minutes after receiving the vaccine."

By Helen Albert, Senior medwireNews Reporter

\section{Reference}

Ann Allergy Asthma Immunol 2012; Advance online publication 